\title{
Effect of the Trace Anomaly on the Cosmological Constant
}

\author{
Jurjen F. Koksma ${ }^{1, \text { * }}$ and Tomislav Prokopec ${ }^{1, \text { 用 }}$ \\ ${ }^{1}$ Institute for Theoretical Physics (ITP) 8 Spinoza Institute, \\ Utrecht University, Postbus 80195, 3508 TD Utrecht, The Netherlands
}

\begin{abstract}
It has been argued that the quantum (conformal) trace anomaly could potentially provide us with a dynamical explanation of the cosmological constant problem. In this paper, however, we show by means of a semiclassical analysis that the trace anomaly does not affect the cosmological constant. We construct the effective action of the conformal anomaly for flat FLRW spacetimes consisting of local quadratic geometric curvature invariants. Counterterms are thus expected to influence the numerical value of the coefficients in the trace anomaly and we must therefore allow these parameters to vary. We calculate the evolution of the Hubble parameter in quasi de Sitter spacetime, where we restrict our Hubble parameter to vary slowly in time, and in FLRW spacetimes. We show dynamically that a Universe consisting of matter with a constant equation of state, a cosmological constant and the quantum trace anomaly evolves either to the classical de Sitter attractor or to a quantum trace anomaly driven one. When considering the trace anomaly truncated to quasi de Sitter spacetime, we find a region in parameter space where the quantum attractor destabilises. When considering the exact expression of the trace anomaly, a stability analysis shows that whenever the trace anomaly driven attractor is stable, the classical de Sitter attractor is unstable, and vice versa. Semiclassically, the trace anomaly does not affect the classical late time de Sitter attractor and hence it does not solve the cosmological constant problem.
\end{abstract}

PACS numbers: 98.80.-k, 04.62.+v, 95.36.+x

\section{INTRODUCTION}

Recent observations have clearly indicated that the expansion of the Universe is accelerating. According to Einstein's general relativity, this can only be realised if the pressure of the dominant component of the current Universe is negative. These observations have triggered a renewed interest in the cosmological constant problem (for recent reviews, see e.g. [1, 2, 3]). What is usually referred to as the "old" cosmological constant problem can be phrased as follows: why is the measured (effective) cosmological constant extremely close to zero?

One approach dealing with the cosmological constant problem is concerned with employing the effective field theory of gravity [4, 5]. Lacking a full quantum theory of gravity, an effective field theory of gravity adopts the following point of view: in order to describe quantum phenomena at very large and cosmologically relevant distances, the precise physics at the shortest distance scales is irrelevant. In other words, the effective field theory of gravity is the low energy limit of quantum gravity. It combines classical general relativity with knowledge of quantum field theory in curved spacetimes $[\underline{6}]$.

In order to describe these long distance effects accurately, one supplements the classical Einstein-Hilbert action with certain additional contributions. One of these additions is the trace anomaly or conformal anomaly which quantum field theories are known to exhibit [6, 7, 8, 9, 10, 11, 12, 13]. If the classical action is invariant under conformal transformations of the metric, the resulting stress-energy tensor is traceless. As an explicit example, one can easily verify that the trace of a massless, conformally coupled scalar field vanishes. In quantum field theory the stress-tensor is promoted to an operator. A careful renormalisation procedure renders its expectation value $\left\langle T^{\mu}{ }_{\nu}\right\rangle$ finite. However, inevitably, the renormalisation procedure results in general in a non-vanishing trace of the renormalised stress-energy tensor. Classical conformal invariance cannot be preserved at the quantum level. Ever since its discovery, the trace anomaly has found many applications in various areas in physics (see e.g. [14]).

An alternative approach to the backreaction problem of quantum fluctuations on the background spacetime deals with quantum fields whose spectrum is nearly flat [15, 16, 17, 18, 19, 20, 21, 22, 23, 24, 25]. Consequently, the spectrum in the infrared is not suppressed and is therefore expected to yield a strong backreaction on the background spacetime. Examples of such fields are the minimally coupled massless scalar and the graviton. 


\section{A. The Connection between the Cosmological Constant and the Trace Anomaly}

Some authors stated that the trace anomaly could have effects on dark energy and the cosmological constant problem [26, 27], whereas it has been argued by other authors that the trace anomaly could potentially provide us with a dynamical explanation of the cosmological constant problem [28, 29, 30, 31, 32, 33. . Broadly speaking, the line of reasoning is as follows (for a more in-depth review, we refer to [33]). The new, conformal degree of freedom is usually parametrised by:

$$
g_{\mu \nu}(x)=e^{2 \sigma(x)} \bar{g}_{\mu \nu}(x)
$$

According to the authors of e.g. [33], the trace anomaly cannot be generated from a local finite term in the action, but rather stems from a non-local effective action that generates the conformal anomaly by variation with respect to the metric [34]. It is this genuine non-locality of the trace anomaly, revealing a large distance effect of quantum physics, that is at the very foundation of its connection with the effective field theory of gravity. One then argues that the new conformal field should dynamically screen the cosmological constant, thus solving the cosmological constant problem.

\section{B. The Semiclassical Approach to the Cosmological Constant and the Trace Anomaly}

The proposal advocated in [33] is very interesting and should be investigated further. Before studying the effect of a new conformal degree of freedom (11), we feel that firstly a proper complete analysis of the dynamics resulting from the effective action of the trace anomaly should be performed. This is what we pursue in this paper.

According to the Cosmological Principle the Universe is homogeneous and isotropic on the largest and cosmologically relevant scales. The CMB measurements [35] constrain the inhomogeneities at order $10^{-4} \sim 10^{-5}$. Moreover, the Universe appears to be spatially flat. Let us make the following observations.

Firstly, the Cosmological Principle dictates the use of the conformally flat FLRW metric $g_{\mu \nu}=a^{2}(\eta) \eta_{\mu \nu}$. Hence, inhomogeneous fluctuations of the metric tensor and in particular of the conformal part of the metric tensor (1) are observed and expected to be small at the largest scales, comparable to the Hubble radius (also in the early Universe).

Secondly, we are led to an essentially semiclassical analysis. The vacuum expectation value of the stress energy tensor resulting in the trace anomaly has been calculated semiclassically. In a semiclassical analysis quantum fluctuations backreact on the background spacetime. Phase transitions aside, quantum fluctuations naturally affect the homogeneous background homogenously. It is a well-known fact that quantum fluctuations can break certain symmetries present in de Sitter [15, 16, 17, 18], e.g. time translation invariance. However, we are not aware of quantum fluctuations breaking the homogeneity and isotropy of the background spacetime.

Finally, if quantum fluctuations compensate for or screen the cosmological constant, we must have $T_{\mu \nu} \propto g_{\mu \nu}$. Let us set: $T_{\mu \nu}=\theta(x) g_{\mu \nu}$. Stress energy conservation and metric compatibility immediately yield: $\nabla_{\mu} \theta(x)=\partial_{\mu} \theta(x)=0$. Hence we conclude that $\theta(x)$ must be a constant: $\theta(x)=\theta_{0}$. Only homogeneous vacuum fluctuations can compensate the cosmological constant. Moreover, $T_{\mu \nu}=\theta_{0} g_{\mu \nu}$ does not break any of the symmetries of a maximally symmetric spacetime $^{1}$. Hence, this form cannot be used to study dynamical backreaction.

The arguments above motivate a semiclassical approach to examining the connection between the cosmological constant and the trace anomaly. Note that we do not consider a new, conformal degree of freedom (1). Hence, we do certainly not exclude any possible effect this (inhomogeneous) conformal degree of freedom might have on the cosmological constant. However, it is plausible that in order to address the link between the cosmological constant and the trace anomaly, a semiclassical analysis suffices.

\section{The Modified Starobinsky Model}

Another application of the conformal anomaly can be found in what has become known as trace anomaly induced inflation: in the absence of a cosmological constant, the trace anomaly could provide us with an effective cosmological constant. Originally, Starobinsky [36] realised that quantum one loop contributions of massless fields can source a de Sitter stage. Subsequently, the theory of trace anomaly induced inflation received significant contributions from [37, 38, 39, 40]. If one includes a cosmological constant, the theory of anomaly induced inflation is plagued by

\footnotetext{
1 Maximally symmetric spacetimes are de Sitter, anti de Sitter and Minkowski spacetime.
} 
instabilities, which we will also come to address. The Modified Starobinsky Model as advocated by [41, 42, 43], takes advantage of these instabilities to account for a graceful exit from inflation. It is argued that supersymmetry breaking changes the degrees of freedom such that it destabilises the quantum anomaly driven attractor and simultaneously stabilises the classical de Sitter attractor.

Improving on e.g. 42, 44], we incorporate matter with a constant equation of state in the Einstein field equations. We argue that it is simply inconsistent not to include matter. Consider the following analogy: if we examine an empty Universe with a cosmological constant only, there is no dynamics and the (00) Einstein equation yields $H^{2}=\Lambda / 3$. Matter drives the dynamics and $H^{2} \neq \Lambda / 3$ can only be realised with $\rho_{\mathrm{M}} \neq 0$.

In the literature, if one solves the trace of the Einstein field equations in a Universe with a cosmological constant and trace anomaly, one solves, however, in reality for the dynamics in a Universe filled with radiation. For it is only radiation with equation of state $w=1 / 3$ that does not contribute to the trace of the Einstein field equation $T_{\text {rad }}=0$. This point has not been included in other papers. We will consider matter with constant but otherwise arbitrary equation of state $w>-1$, and not just (implicitly) radiation with $w=1 / 3$.

\section{Outline}

In this paper we show that the cosmological constant problem cannot be solved by taking account of the trace anomaly alone. The outline of this paper is as follows. In section @ we recall the basics of the conformal anomaly and discuss how to study its effect on the evolution of the Universe by tracing the Einstein field equations.

In section 【II] we derive the conformal anomaly from an effective action in flat homogeneous FLRW spacetimes consisting of local quadratic geometric curvature invariants. Since one usually adds infinite counterterms to cancel the radiative one loop divergences, we do not see any reason why we should exclude adding a Gauss-Bonnet counterterm to cancel the anomaly in flat FLRW spacetimes. Even though this term in the effective action is formally divergent, at the level of the equation of motion it yields a finite result. Hence, the coefficients multiplying the curvature invariants in the trace anomaly are not uniquely specified by the anomaly. The physical coefficient, i.e.: the parameter that can be measured, receives contributions both from the trace anomaly and from possible counterterms cancelling divergences from the underlying (and yet unknown) fundamental theory. This motivates varying the coupling parameters multiplying the curvature invariants in the anomaly. We can thus study all possible effects of the anomaly on the evolution of our Universe.

In section IV we study the evolution of a quasi de Sitter Universe in the presence of matter with constant equation of state, a cosmological constant and the trace anomaly. In quasi de Sitter spacetime we assume, loosely speaking, that the Hubble parameter is a slowly varying function of time. Effectively, we truncate the expression of the exact trace anomaly and discard higher order derivative contributions.

In section $[\nabla$ we generalise our analysis and study the evolution of an FLRW Universe again in the presence of matter with constant equation of state, a cosmological constant and the trace anomaly. We examine the exact trace anomaly and take all higher derivative contributions into account. As the dimensionality of the phase space increases, we must carefully perform a stability analysis of the late time asymptotes.

\section{TRACING THE EINSTEIN FIELD EQUATIONS AND THE TRACE ANOMALY}

\section{A. The Conformal Anomaly in Four Dimensions in FLRW Spacetimes}

The trace anomaly or the conformal anomaly in four dimensions is in general curved spacetimes given by [6, 7, 33$]$ :

$$
T_{\mathrm{Q}} \equiv\left\langle T_{\mu}^{\mu}\right\rangle=b F+b^{\prime}\left(E-\frac{2}{3} \square R\right)+b^{\prime \prime} \square R,
$$

where:

$$
\begin{aligned}
& E \equiv{ }^{*} R_{\mu \nu \kappa \lambda}{ }^{*} R^{\mu \nu \kappa \lambda}=R_{\mu \nu \kappa \lambda} R^{\mu \nu \kappa \lambda}-4 R_{\mu \nu} R^{\mu \nu}+R^{2} \\
& F \equiv C_{\mu \nu \kappa \lambda} C^{\mu \nu \kappa \lambda}=R_{\mu \nu \kappa \lambda} R^{\mu \nu \kappa \lambda}-2 R_{\mu \nu} R^{\mu \nu}+\frac{1}{3} R^{2},
\end{aligned}
$$

where as usual $R_{\mu \nu \kappa \lambda}$ is the Riemann curvature tensor, ${ }^{*} R_{\mu \nu \kappa \lambda}=\varepsilon_{\mu \nu \alpha \beta} R^{\alpha \beta}{ }_{\kappa \lambda} / 2$ its dual, $C_{\mu \nu \kappa \lambda}$ the Weyl tensor and $R_{\mu \nu}$ and $R$ the Ricci tensor and scalar, respectively. Note that $E$ is the Gauss-Bonnet invariant. The general expression for the trace anomaly can also contain additional contributions if the massless conformal field is coupled 
to other long range gauge fields (see e.g. [6] ). Finally, the parameters $b, b^{\prime}$ and $b^{\prime \prime}$ appearing in (2) are dimensionless quantities multiplied by $\hbar$ and are given by:

$$
\begin{aligned}
b & =\frac{1}{120(4 \pi)^{2}}\left(N_{S}+6 N_{F}+12 N_{V}\right) \\
b^{\prime} & =-\frac{1}{360(4 \pi)^{2}}\left(N_{S}+\frac{11}{2} N_{F}+62 N_{V}\right),
\end{aligned}
$$

where $N_{S}, N_{F}$ and $N_{V}$ denote the number of fields of spin $0,1 / 2$ and 1 respectively $(\hbar=1)$. It is important to note that $b>0$ whereas $b^{\prime}<0$ in general. It turns out that the coefficient $b^{\prime \prime}$ is regularisation dependent and is therefore not considered to be part of the true conformal anomaly. We take this into account and study the effect of $b^{\prime \prime}$ on the stability of the solutions we are about to derive. For definiteness, we will assume that these parameters take their Standard Model values: $N_{S}=4, N_{F}=45 / 2$ and $N_{V}=12$. Note if we were to include right-handed neutrinos, $N_{F}=24$. One could also examine the numerical value of the coefficients (4) for the late time Universe. Today's massless particle is just the photon, hence $N_{V}=1, N_{S}=0$ and $N_{F}=0$.

Let us specialise to flat Friedmann-Lemaître-Robertson-Walker or FLRW spacetimes in which the metric is given by $g_{\alpha \beta}=\operatorname{diag}\left(-1, a^{2}(t), a^{2}(t), a^{2}(t)\right)$ where $a(t)$ is the scale factor of the Universe in cosmic time $t$. Recall that a conformal transformation leaves the Weyl tensor invariant. Hence, in FLRW spacetimes $F=0$. Given the FLRW metric one can easily verify that:

$$
\begin{aligned}
R^{2} & =36\left[\dot{H}^{2}+4\left(\dot{H} H^{2}+H^{4}\right)\right] \\
R_{\mu \nu} R^{\mu \nu} & =12\left[\dot{H}^{2}+3\left(\dot{H} H^{2}+H^{4}\right)\right] \\
R_{\mu \nu \kappa \lambda} R^{\mu \nu \kappa \lambda} & =12\left[\dot{H}^{2}+2\left(\dot{H} H^{2}+H^{4}\right)\right] \\
\square R & =-6\left[\dddot{H}+7 \ddot{H} H+4 \dot{H}^{2}+12 \dot{H} H^{2}\right] .
\end{aligned}
$$

Hence, the exact expression for the trace anomaly in FLRW spacetimes in four dimensions reads:

$$
T_{\mathrm{Q}}=4 b^{\prime}\left\{\dddot{H}+7 \ddot{H} H+4 \dot{H}^{2}+18 \dot{H} H^{2}+6 H^{4}\right\}-6 b^{\prime \prime}\left\{\dddot{H}+7 \ddot{H} H+4 \dot{H}^{2}+12 \dot{H} H^{2}\right\} .
$$

To capture the leading order dynamics we work in quasi de Sitter spacetime and allow for a mildly time dependent Hubble parameter:

$$
\epsilon \equiv-\frac{\dot{H}}{H^{2}}=\text { constant } \ll 1,
$$

i.e.: we assume that $\epsilon$ is both small and time independent. This would truncate the trace anomaly up to terms linear in $\dot{H}$ yielding:

$$
T_{\mathrm{Q}}=24 b^{\prime}\left\{3 \dot{H} H^{2}+H^{4}\right\}-72 b^{\prime \prime} \dot{H} H^{2}
$$

We will examine both the exact form of the trace anomaly (6) and its truncated form (8).

Truncating the expression for the trace anomaly is motivated by the following realisation. In general backgrounds we need a non-local effective action to generate the trace anomaly in the equation of motion. The non-locality at the level of the effective action corresponds to an expansion in derivatives at the level of the equation of motion. Generally, higher derivative contributions in an equation of motion have the tendency to destabilise a system unless the initial conditions are highly fine-tuned. Formally, this is known as the theorem of Ostrogradsky and its relevance to Cosmology is outlined, for example, in [45].

Note that when $b^{\prime \prime}=2 b^{\prime} / 3$ the truncated version is exact. We discuss this further in section IVB. Finally, note that although we have truncated equation (6) to obtain (8), equation (8) is still covariant.

\section{B. The Dynamics driven by the Trace Anomaly}

From the Einstein-Hilbert action:

$$
S=S_{\mathrm{EH}}+S_{\mathrm{M}}=\frac{1}{16 \pi G} \int d^{4} x \sqrt{-g}(R-2 \Lambda)+\int d^{4} x \sqrt{-g} \mathcal{L}_{\mathrm{M}},
$$


where:

$$
\mathcal{L}_{\mathrm{M}}=-\frac{1}{2} \partial_{\alpha} \phi(x) \partial_{\beta} \phi(x) g^{\alpha \beta}-\frac{1}{2} m^{2} \phi^{2}(x)-V(\phi(x)),
$$

the Einstein field equations follow as usual as:

$$
R_{\mu \nu}-\frac{1}{2} R g_{\mu \nu}+\Lambda g_{\mu \nu}=8 \pi G T_{\mu \nu}
$$

of which the trace can easily be verified to be:

$$
R-4 \Lambda=-8 \pi G T
$$

where $T=T^{\mu}{ }_{\mu}$. If one considers an empty Universe with a cosmological constant, the (00) Einstein field equation acts as a constraint equation for the Hubble parameter and one simply finds $H^{2}=\Lambda / 3$ as usual. However, for a non-empty Universe, the (00) Einstein field equation becomes a dynamical constraint. The Bianchi identity for the left-hand side of equation (11) straightforwardly results in stress-energy conservation for the right-hand side:

$$
\nabla^{\mu} T_{\mu \nu}=0 .
$$

Because of stress-energy conservation, the (00) and the (ij) components of the Einstein field equations are not independent ${ }^{2}$. Therefore, any linear combination of the (00) and (ij) components of the Einstein field equations combined with stress-energy conservation suffice to describe the time evolution of the Hubble parameter. In particular, the trace equation (12) and stress-energy conservation (13) contain all relevant dynamics for $H$.

Let us set $\phi(x)=\phi_{\mathrm{cl}}(x)+\varphi(x)$ for the quantum field in $S_{\mathrm{M}}$ and require that the classical field obeys the equation of motion. Note that the quantum perturbation $\varphi(x)$ does not obey this equation of motion. We expand in terms of the quantum field and construct the effective action as usual:

$$
\begin{aligned}
\exp \left[i \Gamma\left[\phi_{\mathrm{cl}}\right]\right] & =\exp \left[i S_{\mathrm{M}}\left[\phi_{\mathrm{cl}}\right]\right] \int \mathcal{D} \varphi \exp \left[i\left(\int_{x} \frac{\delta S_{\mathrm{M}}}{\delta \phi_{\mathrm{cl}}(x)} \varphi(x)+\frac{1}{2} \int_{x, y} \frac{\delta^{2} S_{\mathrm{M}}}{\delta \phi_{\mathrm{cl}}(x) \phi_{\mathrm{cl}}(y)} \varphi(x) \varphi(y)+\mathcal{O}\left(\varphi^{3}\right)\right)\right] \\
& =\exp \left[i S_{\mathrm{M}}\left[\phi_{\mathrm{cl}}\right]+i \Gamma_{\mathrm{Q}}\left[\phi_{\mathrm{cl}}\right]\right] .
\end{aligned}
$$

The first contribution to the effective action corresponds to the classical part of the action and $\Gamma_{\mathrm{Q}}\left[\phi_{\mathrm{cl}}\right]$ is the contribution to the effective action taking account of the vacuum fluctuations. The stress-energy tensor now follows as:

$$
T_{\mu \nu}=-\frac{2}{\sqrt{-g}} \frac{\delta}{\delta g^{\mu \nu}} \Gamma\left[\phi_{\mathrm{cl}}\right]=-\frac{2}{\sqrt{-g}} \frac{\delta}{\delta g^{\mu \nu}}\left(S_{\mathrm{M}}\left[\phi_{\mathrm{cl}}\right]+\Gamma_{\mathrm{Q}}\left[\phi_{\mathrm{cl}}\right]\right) \equiv T_{\mu \nu}^{\mathrm{C}}+T_{\mu \nu}^{\mathrm{Q}} .
$$

Hence, there are both classical and quantum contributions to the full stress-energy tensor. Classically, from the equation of motion the scalar field obeys, we have:

$$
\nabla^{\mu} T_{\mu \nu}^{\mathrm{C}}=0
$$

Hence, from (13) we derive:

$$
\nabla^{\mu} T_{\mu \nu}^{\mathrm{Q}}=0 .
$$

Concluding, due to stress-energy conservation at the classical level and for the full stress-energy tensor, we have derived stress-energy conservation for the quantum contributions as well.

Analogously to the classical stress-energy tensor, we can symbolically write: $T_{\nu, \mathrm{Q}}^{\mu}=\left(-\rho_{\mathrm{Q}}, p_{\mathrm{Q}}, p_{\mathrm{Q}}, p_{\mathrm{Q}}\right)$. Combining:

$$
\begin{aligned}
T_{\mathrm{Q}}(t) & =-\rho_{\mathrm{Q}}(t)+3 p_{\mathrm{Q}}(t) \\
\dot{\rho}_{\mathrm{Q}}(t) & =-3 H\left\{\rho_{\mathrm{Q}}(t)+p_{\mathrm{Q}}(t)\right\},
\end{aligned}
$$

\footnotetext{
${ }^{2}$ For example, stress-energy conservation combined with the (00) Einstein field equation straightforwardly yield the (ij) component of the Einstein field equations.
} 
yields:

$$
\frac{d}{d t}\left[a^{4}(t) \rho_{\mathrm{Q}}(t)\right]=-a^{4}(t) H(t) T_{\mathrm{Q}}(t)
$$

We thus find (identical to [37]):

$$
\begin{aligned}
& \rho_{\mathrm{Q}}(t)=-\frac{1}{a^{4}(t)} \int^{t} d \tau a^{4}(\tau) H(\tau) T_{\mathrm{Q}}(\tau) \\
& p_{\mathrm{Q}}(t)=\frac{1}{3}\left(T_{\mathrm{Q}}(t)+\rho_{\mathrm{Q}}(t)\right) .
\end{aligned}
$$

Although in general spacetimes it is not possible to perform this integral, in cosmologically relevant FLRW spacetimes we can [46]. If we consider the exact form of the trace anomaly (6) in flat FLRW spacetimes, we can easily see that $\rho_{\mathrm{Q}}(t)$ should be of the following form:

$$
\rho_{\mathrm{Q}}(t)=b^{\prime}\left[c_{1} \ddot{H} H+c_{2} \dot{H}^{2}+c_{3} \dot{H} H^{2}+c_{4} H^{4}\right]+b^{\prime \prime}\left[c_{5} \ddot{H} H+c_{6} \dot{H}^{2}+c_{7} \dot{H} H^{2}\right] .
$$

Upon inserting this ansatz into equation (19) and equating the contributions at each order, we immediately find:

$$
\rho_{\mathrm{Q}}=2 b^{\prime}\left[-2 \ddot{H} H+\dot{H}^{2}-6 \dot{H} H^{2}-3 H^{4}\right]+3 b^{\prime \prime}\left[2 \ddot{H} H-\dot{H}^{2}+6 \dot{H} H^{2}\right] .
$$

Because $\rho_{\mathrm{Q}}$ can be expressed in a local form, we would like to point out that this yields a local expression for the stressenergy tensor too. Although quantum fluctuations of the conformal field may still act non-locally on the background spacetime, at the classical level the trace anomaly affects the spacetime only locally. Again, when working in quasi de Sitter spacetime where $\epsilon$ is both small and time independent, we can truncate this expression for the quantum density finding:

$$
\rho_{\mathrm{Q}}=-6 b^{\prime}\left[2 \dot{H} H^{2}+H^{4}\right]+18 b^{\prime \prime} \dot{H} H^{2} .
$$

Again, when $b^{\prime \prime}=2 b^{\prime} / 3$, this analysis becomes exact.

In the next sections, we solve the Einstein field equations in FLRW spacetimes for a Universe in the presence of a) a non-zero cosmological constant, b) the trace anomaly as a contribution to the quantum stress-energy tensor and c) matter with constant equation of state $\rho_{\mathrm{M}}=w p_{\mathrm{M}}$, where $w>-1$. We thus consider non-tachyonic matter only, which does not exclude [47, 48], where the effect of a finite period with $w<-1$ is investigated.

The trace anomaly enters the Einstein field equation naturally in the trace equation (12). We can thus study the effect of the trace anomaly on the evolution of a Universe with a cosmological constant and matter. Hence, the trace equation of the Einstein field equations is given by:

$$
R-4 \Lambda=-8 \pi G\left\{T_{\mathrm{Q}}+T_{\mathrm{M}}\right\}
$$

where

$$
T_{\mathrm{M}}=-\rho_{\mathrm{M}}+3 p_{\mathrm{M}}=\rho_{\mathrm{M}}(3 \omega-1) .
$$

Now, we can employ the (00) Einstein field equation from (111) to express $\rho_{\mathrm{M}}$ in terms of $\rho_{\mathrm{Q}}$ yielding:

$$
9(1+\omega) H^{2}(t)+6 \dot{H}(t)-3(1+\omega) \Lambda=-8 \pi G\left[T_{\mathrm{Q}}+(1-3 \omega) \rho_{\mathrm{Q}}\right] .
$$

The above equation governs the dynamics for the Hubble parameter that we will solve in various interesting cases. For the anomalous trace we can either take the exact expression (6) containing higher derivatives of the Hubble parameter or its truncated version (8). Likewise, for the quantum density we can either insert the full expression (22) or the truncated one (23). The reason for truncating the expression for the quantum trace and density as outlined above is the realisation that higher derivative contributions in an equation of motion generally have the tendency to destabilise a system.

In the literature (see e.g. [43]), one only has considered an empty Universe with a cosmological term and trace anomaly. In reality, one solved for a radiation dominated Universe, for only radiation $(\omega=1 / 3)$ does not contribute to the trace of the Einstein field equations. We incorporate matter with constant equation of state parameter $\omega$. 
Independently on whether one truncates the expressions for the anomalous trace or quantum density, one can solve for the asymptotes yielding the late time behaviour. Setting all time derivatives of the Hubble parameter equal to zero, one can easily solve for the two late time constants:

$$
\left(H_{0}^{\mathrm{C}, \mathrm{A}}\right)^{2}=\frac{-1 \pm \sqrt{1+64 \pi G b^{\prime} \Lambda / 3}}{32 \pi G b^{\prime}}
$$

Here, $H_{0}^{\mathrm{C}}$ turns out to be the classical de Sitter attractor, whereas $H_{0}^{\mathrm{A}}$ is a new, quantum anomaly driven attractor. This result for the late time constants is identical to the case where matter is absent [42]. We can write the above expression in a somewhat more convenient form by defining the dimensionless parameter $\lambda$ :

$$
\lambda=\frac{G \Lambda}{3},
$$

that sets the scale for the cosmological constant $\Lambda$. In the current epoch, $\lambda$ is extremely small, which allows us to expand (27) finding ${ }^{3}$ :

$$
\begin{aligned}
H_{0}^{\mathrm{C}} & =\sqrt{\frac{\Lambda}{3}}\left[1-8 \pi b^{\prime} \lambda\right] \\
H_{0}^{\mathrm{A}} & =\sqrt{\frac{-1}{16 \pi G b^{\prime}}-\frac{\Lambda}{3}} .
\end{aligned}
$$

In the absence of a cosmological constant, the trace anomaly can thus provide us with an inflationary scenario which has already been appreciated by [36, 37, 39, 40]. Finally, note these asymptotes are independent of $b^{\prime \prime}$.

\section{THE EFFECTIVE ACTION GENERATING THE TRACE ANOMALY}

The authors of [33] are correct in saying that the conformal anomaly cannot be generated by a finite effective action built out of local quadratic geometric curvature invariants only. We show that the trace anomaly can be generated by an infinite effective action in flat FLRW spacetimes, that consists only of local quadratic geometric curvature invariants. Although infinite at the level of the effective action, we generate a finite on shell contribution. We first write the trace anomaly in conformal time $d t=a(\eta) d \eta$ such that the full conformal anomaly (6) reads:

$$
T_{\mathrm{Q}}=24 b^{\prime}\left(\frac{a^{\prime \prime}}{a^{3}}\left(\frac{a^{\prime}}{a^{2}}\right)^{2}-\left(\frac{a^{\prime}}{a^{2}}\right)^{4}\right)-6\left(b^{\prime \prime}-2 b^{\prime} / 3\right)\left(\frac{a^{\prime \prime \prime \prime}}{a^{5}}-4 \frac{a^{\prime \prime \prime}}{a^{4}} \frac{a^{\prime}}{a^{2}}-3\left(\frac{a^{\prime \prime}}{a^{3}}\right)^{2}+6 \frac{a^{\prime \prime}}{a^{3}}\left(\frac{a^{\prime}}{a^{2}}\right)^{2}\right) .
$$

Here, dashes denote conformal time derivatives. In general, the trace of a stress-energy tensor can be written as:

$$
T=-\frac{2}{\sqrt{-g}} g^{\mu \nu} \frac{\delta S}{\delta g^{\mu \nu}}=\frac{4}{a^{3}(\eta) V} \frac{\delta S}{\delta a} .
$$

Here, $V$ is the (spatial) volume. The correct effective action $\Gamma_{\text {an }}$ that generates the trace anomaly in spatially flat FLRW spacetimes is given by:

$$
\Gamma_{\mathrm{an}}=\int d^{D} x \sqrt{-g}\left\{\beta_{\mathrm{D}} E-12\left(b^{\prime \prime}-2 b^{\prime} / 3\right) R^{2}\right\}
$$

where

$$
\beta_{\mathrm{D}}=b^{\prime} \frac{1}{(D-4)}+\beta_{0}
$$

\footnotetext{
3 Note the nomenclature in the literature is somewhat misleading. Rather than calling 29a the classical de Sitter attractor, it would be more natural to denote it with the quantum corrected classical attractor. Hence, the quantum attractor 29b should preferably be denoted by anomaly driven attractor or Planck scale attractor. We will nevertheless adopt the nomenclature existing in the literature.
} 
Here $D$ is the dimension of the spacetime and $\beta_{0}$ is an undetermined finite (and physically irrelevant) constant. Only after variation we let $D \rightarrow 4$. The numerical factors in (32) are chosen in accordance with the trace of the Einstein field equations, i.e.: the trace of the variation:

$$
g^{\mu \nu} \frac{\delta}{\delta g^{\mu \nu}} S=g^{\mu \nu} \frac{\delta}{\delta g^{\mu \nu}}\left[S_{\mathrm{EH}}+\Gamma_{\mathrm{an}}\right]=0,
$$

indeed yields:

$$
R-4 \Lambda=-8 \pi G T
$$

The effective action (32) merits some clarifying remarks. Firstly, the variation of $R^{2}$ straightforwardly yields the $\square R$ term in the conformal anomaly. This term is not unique for one could have equally well taken $R^{\mu \nu} R_{\mu \nu}$ into account ${ }^{4}$. Secondly, note that the local effective action depends solely on the scale factor. If one were to rewrite this effective action covariantly in terms of the full metric, the effective action would become non-local [8, 34], also see [49, 50]. It is well-known that the effective action of the conformal factor can be written in a local form [46, 51]. However, we have been able to rewrite this expression in terms of the Gauss-Bonnet invariant multiplying an infinite constant. Finally, note that the coefficient (33) diverges in four dimensions. However, the Gauss-Bonnet invariant in $D$ dimensions reads:

$$
E=\left(\frac{a^{\prime}}{a^{2}}\right)^{4}[(D-8)(D-3)(D-2)(D-1)]+\frac{a^{\prime \prime}}{a^{3}}\left(\frac{a^{\prime}}{a^{2}}\right)^{2}[4(D-3)(D-2)(D-1)]
$$

which can be determined from the $D$ dimensional generalisations of equation (5). Note:

$$
-\frac{2}{\sqrt{-g}} g^{\mu \nu} \frac{\delta}{\delta g^{\mu \nu}} \int d^{D} x \sqrt{-g} E=D(D-4) E
$$

Clearly, only in four dimensions the Gauss-Bonnet term corresponds to a total derivative that can be neglected. Hence, we choose the divergent coefficient $\beta_{\mathrm{D}}$ such that the factor $(D-4)$ cancels yielding a non-vanishing and finite contribution at the level of the equation of motion when $D \rightarrow 4$. The contribution from $\beta_{0}$ is identically zero when we let $D \rightarrow 4$. Although divergent in the action, we have shown that at the level of the equation of motion the term involving $\beta_{\mathrm{D}}$ yields a finite contribution. All physical measurements are performed on shell, hence we cannot exclude a counterterm of the form $\beta_{\mathrm{D}} E$ in the effective action.

This procedure can be debated. In the literature one usually prefers a finite effective action. We abandon this assumption and require a finite equation of motion only. We cannot think of a physical measurement that distinguishes the two approaches and therefore we argue that one should consider and examine all possible effects the counterterms might have on the trace anomaly.

In homogeneous cosmology, $R^{2}$ and $E$ are the only local quadratic geometric curvature invariants ${ }^{5}$. Divergences up to one loop in perturbative quantum gravity can be cancelled only by counterterms of the form $R^{2}$ and $E$. The physical coefficients multiplying $R^{2}$ and $E$ in the renormalised one loop effective action receive contributions which one can write as:

$$
\begin{aligned}
\alpha_{\text {phys }} R^{2} & =\left[\alpha_{\text {anom }}(m)+\alpha_{\mathrm{ct}}\right] R^{2} \\
\beta_{\text {phys }} E & =\left[\beta_{\text {anom }}(m)+\beta_{\mathrm{ct}}\right] E,
\end{aligned}
$$

where $\alpha_{\text {anom }}(m)$ is the mass dependent finite contribution from the trace anomaly, where $m=\left\{m_{i}\right\}$ denotes the mass of the particles $i$. It is in principle uniquely defined by the requirement that $\alpha_{\text {anom }} \rightarrow 0$ as $m \rightarrow \infty$. Similarly, $\beta_{\text {anom }}(m)$ is the (infinite) anomalous contribution determined by the requirement that $\beta_{\text {anom }} \rightarrow 0$ as $m \rightarrow \infty$. These requirements in principle fix $\beta_{\text {anom }}(m)$ and $\alpha_{\text {anom }}(m)$ uniquely. While there is agreement in the literature on the value of $\alpha_{\text {anom }}(m)$ (also used in this paper), disagreement exists on the value of $\beta_{\text {anom }}(m)$. Therefore we leave it unspecified. The contributions $\alpha_{\text {ct }}$ and $\beta_{\text {ct }}$ correspond to the parts of the counterterms that remain when eventual one loop divergences are cancelled ${ }^{6}$. It is however only the sum of these terms, yielding $\alpha_{\text {phys }}$ and $\beta_{\text {phys }}$, that is

\footnotetext{
${ }^{4}$ We can easily split $R^{\mu \nu} R_{\mu \nu}$ in an $R^{2}$ contribution and a Gauss-Bonnet term, that yields a vanishing surface term in four dimensions.

${ }^{5}$ Because $F=0$, we can express $R_{\mu \nu \kappa \lambda} R^{\mu \nu \kappa \lambda}$ in terms of $R_{\mu \nu} R^{\mu \nu}$ and $R^{2}$. Hence, $R^{2}$ and $E$ are linearly independent. Note furthermore that if one investigates inhomogeneities in the Universe, one measures (statistical) correlation functions. They are translation invariant as a consequence of the symmetry of the vacuum state and therefore respect the symmetry of the background spacetime.

${ }^{6}$ For a calculation involving anomaly calculations around Minkowski spacetime, we refer to [52, 53]. For the calculation of the $\alpha_{\text {anom }}(m)$ function for a scalar field in de Sitter spacetime, we refer to [6].
} 
physical, i.e. measurable [24, 25]. Since these parameters have not been measured, we cannot simply assume that the coefficients in the trace anomaly are just given by (4). We should allow these coefficients to vary in order to examine the full effect of the trace anomaly on the evolution of our FLRW Universe. This is precisely what we pursue in the following sections.

\section{THE TRACE ANOMALY IN QUASI DE SITTER SPACETIME}

In this section we work in quasi de Sitter spacetime, where we treat $\epsilon$ as a small and time independent constant which allows us to neglect higher order derivative contributions. The reason for discarding higher order derivative contributions is that they tend to destabilise a dynamical system, formally known as the theorem of Ostrogradsky [45].

We have to distinguish two cases separately. In the spirit of [33], the numerical value of the parameter $b^{\prime \prime}$ occurring in the trace anomaly is not fixed because it is a regularisation scheme dependent parameter. Generally, however, we cannot exclude the presence of this term and we therefore allow it to take different values. First, we allow for an unrestricted value of $b^{\prime \prime}$ and secondly we set $b^{\prime \prime}=2 b^{\prime} / 3$. This case is particularly interesting as this value of $b^{\prime \prime}$ sets the total coefficient multiplying the $\square R$ contribution in the trace anomaly to zero.

\section{A. Case I: unrestricted value of $b^{\prime \prime}$}

We thus insert the truncated expression for the trace anomaly (8) and the quantum density (23) into the Einstein field equation (26). This yields:

$$
9(1+\omega) H^{2}(t)+6 \dot{H}(t)-3(1+\omega) \Lambda=-8 \pi G\left[\left\{12 b^{\prime}(5+3 \omega)-54 b^{\prime \prime}(1+\omega)\right\} \dot{H} H^{2}+18 b^{\prime}(1+\omega) H^{4}\right] .
$$

This differential equation can be solved exactly. Separation of variables yields:

$$
t-t^{\prime}=\frac{1}{(1+\omega)} \int_{H\left(t^{\prime}\right)}^{H(t)} d H \frac{2+\alpha H^{2}}{\Lambda-3 H^{2}-48 \pi G b^{\prime} H^{4}},
$$

where $\alpha$ is conveniently defined as:

$$
\alpha=8 \pi G\left\{4 b^{\prime}(5+3 \omega)-18 b^{\prime \prime}(1+\omega)\right\} .
$$

One can perform the integral in terms of logarithms where one has to take the signs of the occurring parameters carefully into account. The integral above gives:

$$
\begin{aligned}
t-t^{\prime}=\frac{1}{-48 \pi G b^{\prime}(1+\omega)\left\{\left(H_{0}^{\mathrm{A}}\right)^{2}-\left(H_{0}^{\mathrm{C}}\right)^{2}\right\}} & {\left[-\frac{1+\frac{1}{2} \alpha\left(H_{0}^{\mathrm{A}}\right)^{2}}{H_{0}^{\mathrm{A}}}\left\{\log \left(\frac{H(t)+H_{0}^{\mathrm{A}}}{H(t)-H_{0}^{\mathrm{A}}}\right)-\log \left(\frac{H\left(t^{\prime}\right)+H_{0}^{\mathrm{A}}}{H\left(t^{\prime}\right)-H_{0}^{\mathrm{A}}}\right)\right\}\right.} \\
+ & \left.\frac{1+\frac{1}{2} \alpha\left(H_{0}^{\mathrm{C}}\right)^{2}}{H_{0}^{\mathrm{C}}}\left\{\log \left(\frac{H(t)+H_{0}^{\mathrm{C}}}{H(t)-H_{0}^{\mathrm{C}}}\right)-\log \left(\frac{H\left(t^{\prime}\right)+H_{0}^{\mathrm{C}}}{H\left(t^{\prime}\right)-H_{0}^{\mathrm{C}}}\right)\right\}\right] .
\end{aligned}
$$

Note the asymptotes of this analytic solution coincide with the asymptotes obtained earlier in (29) as expected.

In figure 1 we numerically calculate the dynamics of the Hubble parameter for various initial conditions. The two asymptotes divide this graph into three distinct regions that are not connected for finite time evolution. The region bounded by the two asymptotes contains initial conditions for $H(t)$ such that $H(t)$ grows for late times towards $H_{0}^{\mathrm{A}}$ and initial conditions such that $H(t)$ asymptotes to the de Sitter attractor $H_{0}^{\mathrm{C}}$. In figures 2 and 3 , we examine whether our approximation that $\epsilon$ is both small and a constant is valid for calculating the dynamics. In figure 2 one can clearly see that $\epsilon \ll 1$ for late times. If $\dot{\epsilon}=0$, we should have $\dot{\epsilon} /(H \epsilon) \ll 1$, an assumption that is violated as depicted in figure 3. However, already for a classical cosmological constant dominated Universe with matter, a similar violation occurs.

Furthermore, note the existence of a branching point, an initial condition for $H(t)$ such that for $H(0)>H_{\mathrm{BP}}$ the Hubble parameter asymptotes to the quantum attractor $H_{0}^{\mathrm{A}}$ and for $H(0)<H_{\mathrm{BP}}$ the Hubble parameter decreases to the classical de Sitter attractor $H_{0}^{\mathrm{C}}$. When rewriting equation of motion (39) in terms of $\epsilon$ and noting that $\epsilon$ should diverge exactly at the branching point, one finds:

$$
H_{\mathrm{BP}}=\frac{1}{\sqrt{8 \pi G\left\{9(1+\omega) b^{\prime \prime}-2(5+3 \omega) b^{\prime}\right\}}} .
$$




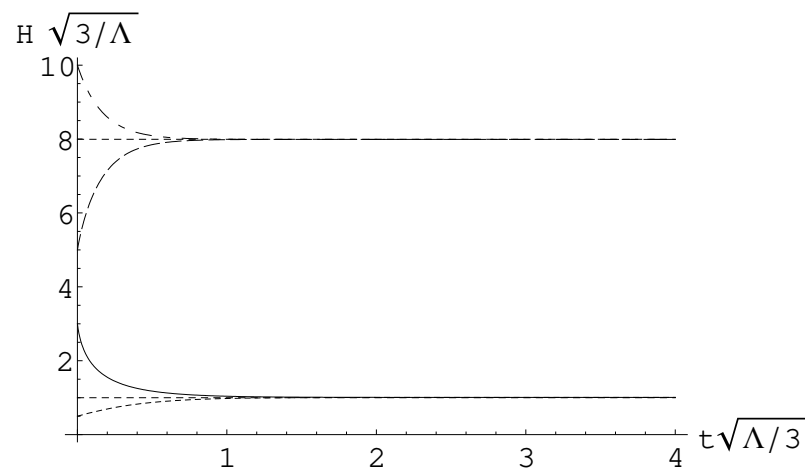

Figure 1: Dynamics of the Hubble parameter in quasi de Sitter spacetime in the presence of a non-zero cosmological constant, the trace anomaly, and matter $(\omega=0)$. Depending on the initial conditions, the Hubble parameter evolves to either the classical de Sitter or the quantum anomaly driven attractor. We have used $\lambda=1 / 50$, $b^{\prime}=-0.015$ (Standard Model value) and $b^{\prime \prime}=0$.

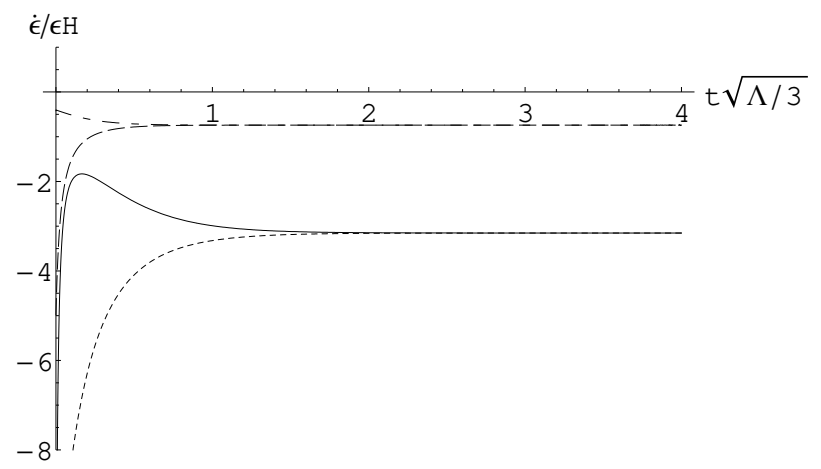

Figure 3: Validity of the assumption $\dot{\epsilon}=0$. For the various initial conditions in figure 1, we have calculated $\dot{\epsilon} /(H \epsilon)$. Clearly, this condition is violated at all times.

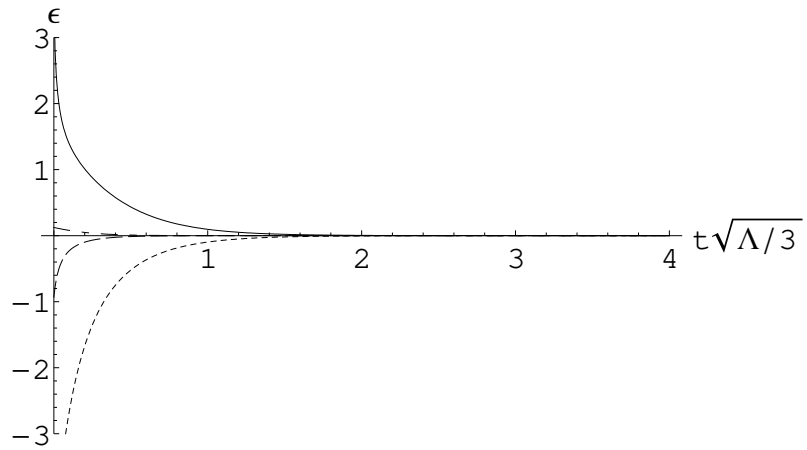

Figure 2: Validity of the assumption $\epsilon \ll 1$. For the various initial conditions in figure 1 one can clearly see that this approximation is well justified.

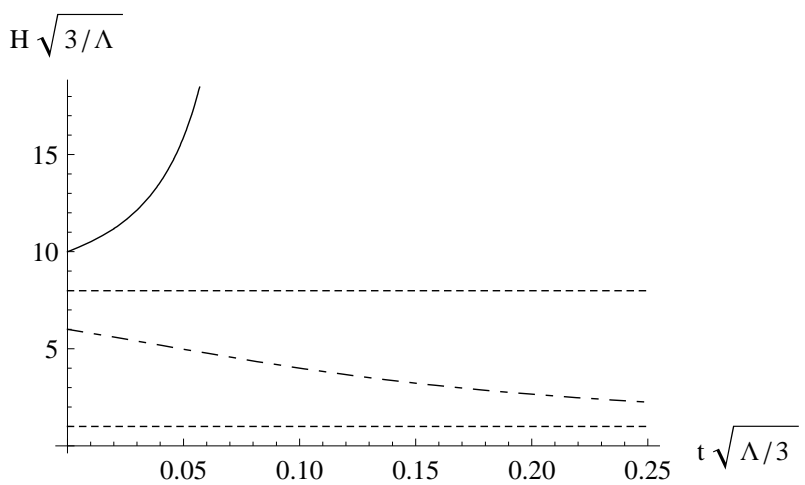

Figure 4: Instability of the quantum anomaly driven attractor in quasi de Sitter spacetimes. We have used $\lambda=1 / 50, \omega=1 / 3, b^{\prime \prime}=7 b^{\prime} / 6<5 b^{\prime} / 6$.

We probe the dependence on scale by changing the numerical value attached to $\lambda$ in equation (28). If we decrease $\lambda$, then also $\Lambda$ decreases which results in a smaller $H_{0}^{\mathrm{C}}$. Also, it turns out that both of the asymptotes are already reached much faster. This improves the validity of the assumption $\epsilon \ll 1$, whereas the assumption $\dot{\epsilon} /(H \epsilon) \ll 1$ is still seriously violated at all times.

Let us study the analytical solution (42) more closely. In particular, it is interesting to derive the high and low energy limits of this solution. Of course, one naively expects in the high or low energy limit to flow towards the quantum or classical attractor, respectively. However, the analysis turns out to be somewhat more subtle. We will show that under a certain condition, the quantum anomaly driven attractor becomes unstable. Although solution (42) looks complicated at first glance, it simplifies when defining:

$$
\begin{aligned}
\Omega & =3(1+\omega)\left[1+32 \pi b^{\prime} \lambda\right] \\
A & =\frac{1+\frac{1}{2} \alpha\left(H_{0}^{\mathrm{A}}\right)^{2}}{H_{0}^{\mathrm{A}}} \\
B & =\frac{1+\frac{1}{2} \alpha\left(H_{0}^{\mathrm{C}}\right)^{2}}{H_{0}^{\mathrm{C}}} .
\end{aligned}
$$


We define the initial conditions at $t^{\prime}$ as:

$$
\begin{aligned}
& c_{1}=\log \left(\frac{H\left(t^{\prime}\right)+H_{0}^{\mathrm{A}}}{\left|H\left(t^{\prime}\right)-H_{0}^{\mathrm{A}}\right|}\right) \\
& c_{2}=\log \left(\frac{H\left(t^{\prime}\right)+H_{0}^{\mathrm{C}}}{\left|H\left(t^{\prime}\right)-H_{0}^{\mathrm{C}}\right|}\right) .
\end{aligned}
$$

Note that $\Omega>1$, generically. With these definitions, equation (42) reduces to:

$$
\Omega\left(t-t^{\prime}\right)=-A\left[\log \left(\frac{H(t)+H_{0}^{\mathrm{A}}}{\left|H(t)-H_{0}^{\mathrm{A}}\right|}\right)-c_{1}\right]+B\left[\log \left(\frac{H(t)+H_{0}^{\mathrm{C}}}{\left|H(t)-H_{0}^{\mathrm{C}}\right|}\right)-c_{2}\right],
$$

In the high energy limit, we set:

$$
\delta(t)=\frac{H(t)-H_{0}^{\mathrm{A}}}{H_{0}^{\mathrm{A}}},
$$

such that $\delta(t) \ll 1$. Equation (45) thus modifies to:

$$
\Omega\left(t-t^{\prime}\right)=-A\left[\log \left(\frac{2+\delta(t)}{|\delta(t)|}\right)-c_{1}\right]+B\left[\log \left(\frac{1+\delta(t)+H_{0}^{\mathrm{C}} / H_{0}^{\mathrm{A}}}{\left|1+\delta(t)-H_{0}^{\mathrm{C}} / H_{0}^{\mathrm{A}}\right|}\right)-c_{2}\right]
$$

We can expand the second logarithm making use of $\lambda \ll 1$. The leading order contribution (in $\delta(t))$ is given by the denominator in the logarithm, because this term diverges as $\delta(t)$ approaches zero. We can thus exponentiate the equation and solve for the Hubble parameter:

$$
\left|H_{>}(t)-H_{0}^{\mathrm{A}}\right|=2 H_{0}^{\mathrm{A}} \exp \left[\frac{\Omega}{A}\left(t-t^{\prime}+\Delta t_{>}\right)\right]
$$

where the time shift $\Delta t_{>}$is given by:

$$
\Omega \Delta t_{>}=B\left(c_{2}-2 \frac{H_{0}^{\mathrm{C}}}{H_{0}^{\mathrm{A}}}\right)-A c_{1} .
$$

As $\Omega>0$, this solution converges whenever $A<0$. This provides a stability condition on $b^{\prime \prime}$ in terms of $b^{\prime}$ and $\lambda$. The quantum anomaly driven attractor is stable, whenever the following inequality is satisfied:

$$
b^{\prime \prime}>\frac{2}{9} b^{\prime} \frac{4+3 \omega+16 \pi \lambda b^{\prime}(5+3 \omega)}{(1+\omega)\left(1+16 \pi \lambda b^{\prime}\right)} .
$$

In figure 4, we have numerically calculated the evolution of the Hubble parameter in a radiation dominated Universe when this inequality is not satisfied. We used $b^{\prime \prime}=7 b^{\prime} / 6<5 b^{\prime} / 6$. For initial conditions above the attractor, the Hubble parameter increases to even higher energies, whereas for initial conditions below the quantum attractor, the Hubble parameter evolves towards the classical attractor. Hence even in quasi de Sitter spacetimes, physically questionable solutions occur. However, note that when $b^{\prime \prime}=2 b^{\prime} / 3$, the specific case under consideration in subsection IVB the above inequality is satisfied.

When the above inequality is satisfied, the Hubble parameter in the high energy limit decays exponentially towards the quantum anomaly driven attractor, where some "frequency dependence" through $\Omega / A$ and a time shift $\Delta t_{>}$can be recognised. The time shift can without observational consequences be absorbed in the initial time $t^{\prime}$.

The low energy limit reveals less surprising behaviour. Here, we set:

$$
\tilde{\delta}(t)=\frac{H(t)-H_{0}^{\mathrm{C}}}{H_{0}^{\mathrm{C}}}
$$

and $\tilde{\delta}(t) \ll 1$. Again we use $\lambda \ll 1$ in order to capture the leading order dynamics. This yields:

$$
\left|H_{<}(t)-H_{0}^{\mathrm{C}}\right|=2 H_{0}^{\mathrm{C}} \exp \left[-\frac{\Omega}{B}\left(t-t^{\prime}+\Delta t_{<}\right)\right] \text {. }
$$




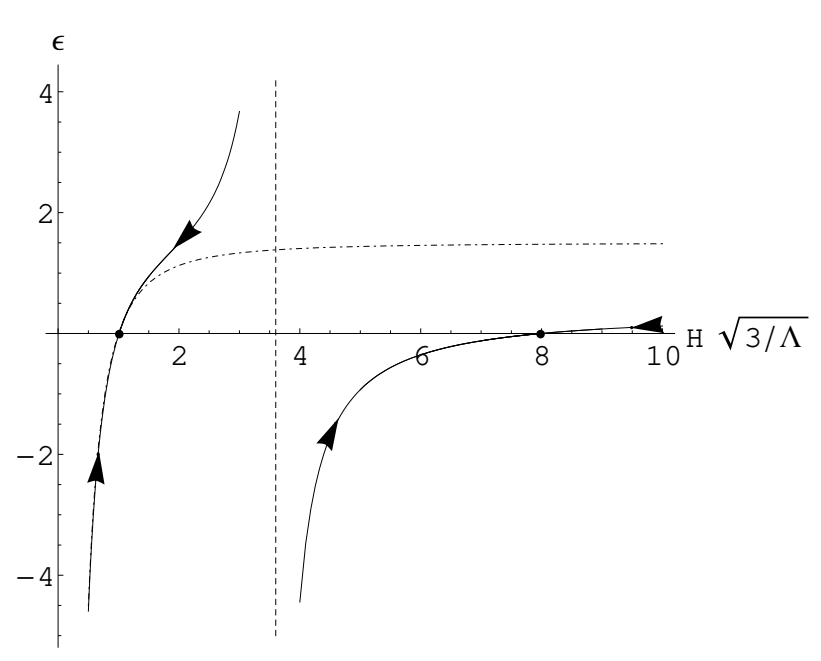

Figure 5: Phase space flow in quasi de Sitter spacetime. The phase space consists of two lines. The flow, indicated by the arrows, is towards the classical or quantum attractor represented by two dots. In this regime, both attractors are stable. The vertical dashed line indicates the branching point and the dashed-dotted line the classical evolution. We have used $\omega=0, \lambda=1 / 50, b^{\prime}=-0.015$ (Standard Model value) and $b^{\prime \prime}=0$.

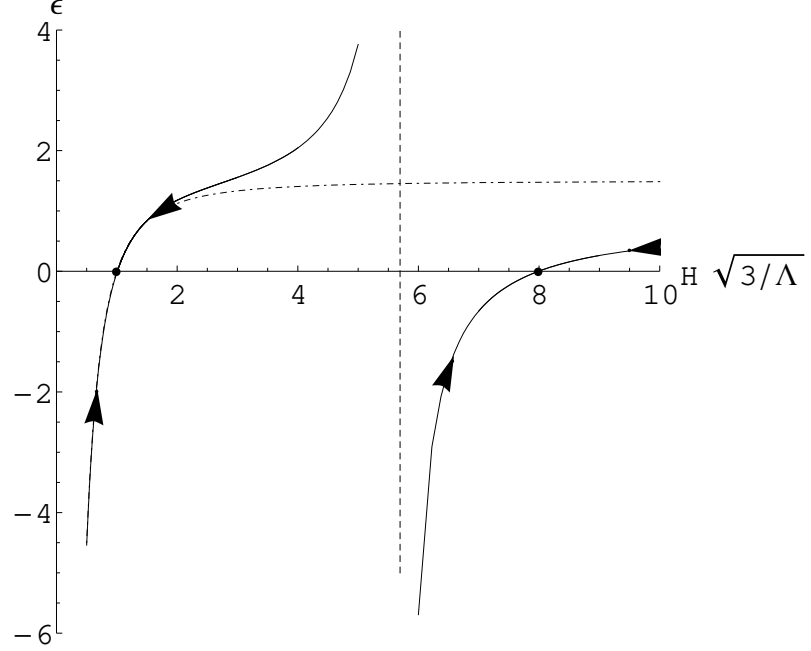

Figure 6: Phase space flow in quasi de Sitter spacetime for $b^{\prime \prime}=2 b^{\prime} / 3$ such that the $\square R$ does not contribute in the trace anomaly. Qualitatively, the dynamics does not change when compared to figure 5. Again, both attractors are stable. Apart from $b^{\prime \prime}$, the value of the parameters are identical to figure 5

Because $B>0$, this solution converges. In the low energy limit the time shift $\Delta t_{<}$is slightly different as compared to (49):

$$
\Omega \Delta t_{<}=B c_{2}-A\left(c_{1}-2 \frac{H_{0}^{\mathrm{C}}}{H_{0}^{\mathrm{A}}}\right) .
$$

Finally, we examine the phase space flow in quasi de Sitter spacetime. In figure 5 we show a parametric plot of $H$ versus $\epsilon$. The phase space basically consists of two lines. The phase space flow is towards either the classical or the quantum anomaly driven attractor as indicated by the arrows. Note that we have chosen both attractors to be stable. Furthermore, we also include the branching point (43) and the classical evolution, that is, the evolution of a Universe with $b^{\prime \prime}=b^{\prime}=0$. As expected, the flow is towards the classical attractor in this case. Although the analysis performed above is for generic values of $b^{\prime \prime}$, we set it to zero in figure 5 and $b^{\prime}$ takes its Standard Model value.

\section{B. Case II: $\mathbf{b}^{\prime \prime}=2 \mathbf{b}^{\prime} / 3$}

As indicated earlier, we must consider the case when $b^{\prime \prime}=2 b^{\prime} / 3$ separately because in this particular case the total coefficient in front of the $\square R$ contribution to the trace anomaly vanishes. All higher derivative contributions precisely cancel and also the $\dot{H}^{2}$ contribution happens to cancel, such that we find ourselves immediately situated in quasi de Sitter spacetime. Albeit a simple case, we do take the full trace anomaly into account.

The analytic solution obtained in (42) still applies and moreover, it becomes exact. The branching point is still given by equation (43) for which we just have to insert $b^{\prime \prime}=2 b^{\prime} / 3$. Clearly, in figure 6 one can see that qualitatively the dynamics has not changed compared to figure 5. The branching point has shifted somewhat to the right, and the way in which the Hubble parameter approaches its two late time asymptotes differs. However, the important features of figure 5, i.e.: two stable attractors, the occurrence of a branching point and the shape and dimension of the phase space, do not change.

\section{THE TRACE ANOMALY IN FLRW SPACETIMES}

We turn our attention to solving the full trace equation (26), where we truncate the expression neither for the anomalous trace (6) nor for the quantum density (22). Obviously, we cannot solve this equation analytically, for it 


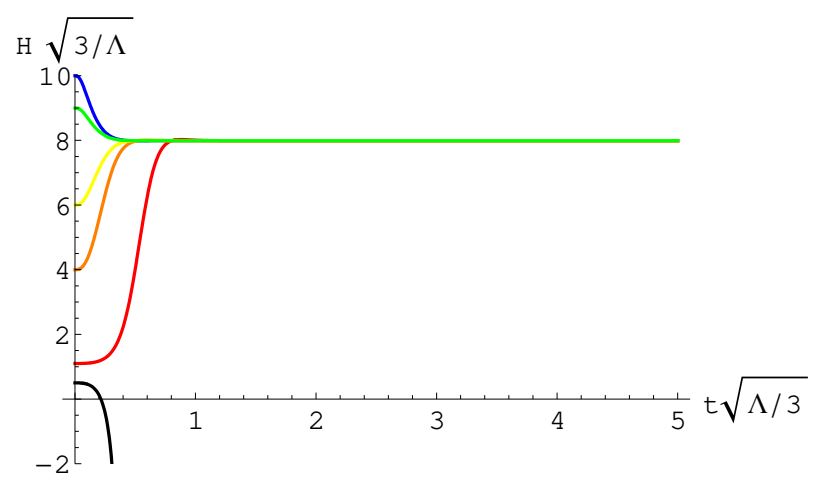

Figure 7: Dynamics of the Hubble parameter taking the full trace anomaly into account. We took $b^{\prime \prime}=0$ such that $b^{\prime \prime}-2 b^{\prime} / 3>0$ yielding an unstable classical attractor. We have used $\omega=0, \lambda=1 / 50, b^{\prime}=-0.015$ (Standard Model value).

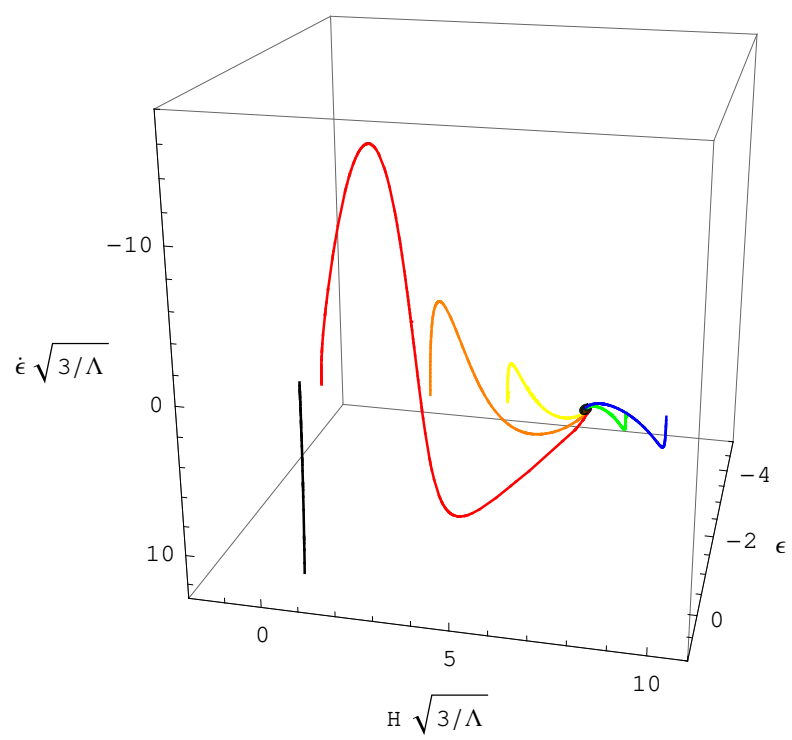

Figure 8: Parametric phase space plot for figure 7 in which the classical attractor is unstable. We have indicated the quantum anomaly driven attractor as a small black sphere.

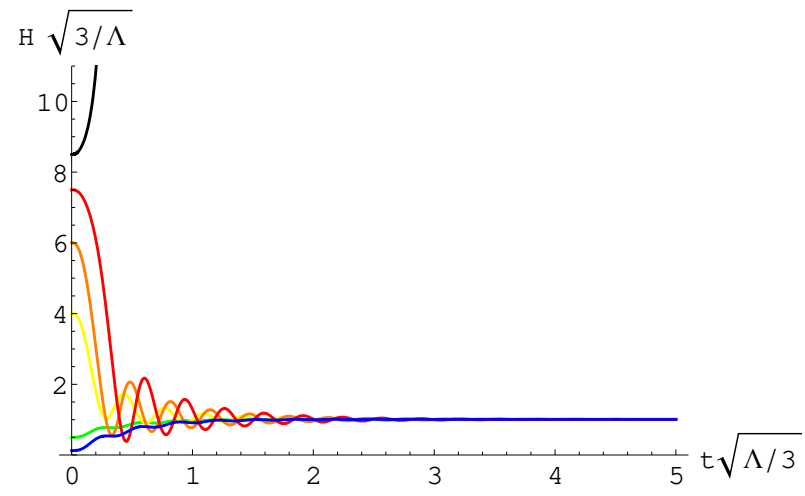

Figure 9: Dynamics of the Hubble parameter taking the full trace anomaly into account. We took $b^{\prime \prime}=b^{\prime}$ such that $b^{\prime \prime}-2 b^{\prime} / 3<0$ yielding a stable classical attractor. We have used $\omega=0, \lambda=1 / 50, b^{\prime}=-0.015$ (Standard Model value). Clearly, the classical attractor is under-damped, resulting in various oscillations around $H_{0}^{\mathrm{C}}$.

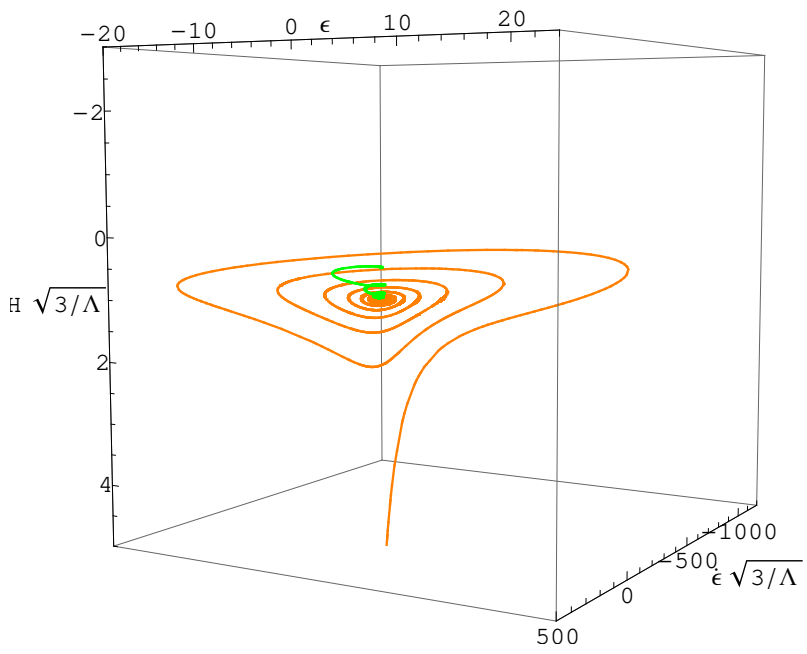

Figure 10: Parametric phase space plot for figure 9 in which the classical attractor is stable. Because of underdamping, one spirals towards the classical attractor. For clarity, we have only included the flow for two initial conditions $H(0)=\sqrt{3 / \Lambda} / 2$ and $H(0)=6 \sqrt{3 / \Lambda}$. The twister like structure is clearly visible. Qualitatively, the phase space flow resulting from other initial conditions is identical.

contains all higher order derivative contributions, which forces us to rely on numerical methods.

Firstly, we note that the asymptotes (29) do not change by including the higher derivative contributions. Keeping Ostrogradsky's theorem in mind, we expect to incur all kinds of issues related to the stability of our system and asymptotes in particular. It is therefore essential to perform a stability analysis for small perturbations $\delta H(t)$ around both of the asymptotes. We insert:

$$
H(t)=H_{0}^{\mathrm{C}, \mathrm{A}}+\delta H(t)
$$

in equation (26), where $H_{0}^{\mathrm{C}, \mathrm{A}}$ can either denote the classical or the quantum attractor. For the small perturbations around these attractors we make the ansatz $\delta H(t)=c \exp [\xi t]$. We linearise the trace equation finding the characteristic 
equation from which we determine the eigenvalues $\xi$ of our system:

$$
\begin{aligned}
2(\mu-3 \nu / 2) \xi^{3}+6(\mu-3 \nu / 2)(2+\omega) H_{0}^{\mathrm{C}, \mathrm{A}} \xi^{2}+\left(\{6 \mu(5+3 \omega)-27 \nu(1+\omega)\}\left(H_{0}^{\mathrm{C}, \mathrm{A}}\right)^{2}-3\right) \xi & \\
& +9\left(4 \mu\left(H_{0}^{\mathrm{C}, \mathrm{A}}\right)^{2}-1\right)(1+\omega) H_{0}^{\mathrm{C}, \mathrm{A}}=0,
\end{aligned}
$$

where $\mu=-8 \pi G b^{\prime}$ and $\nu=-8 \pi G b^{\prime \prime}$. Remarkably, the solutions of this third order equation are simple:

$$
\begin{aligned}
& \xi^{(1)}=-3 H_{0}^{\mathrm{C}, \mathrm{A}}(1+\omega) \\
& \xi^{(2)}=-\frac{3 H_{0}^{\mathrm{C}, \mathrm{A}}}{2}+\sqrt{\Delta} \\
& \xi^{(3)}=-\frac{3 H_{0}^{\mathrm{C}, \mathrm{A}}}{2}-\sqrt{\Delta},
\end{aligned}
$$

where:

$$
\Delta=-\frac{3}{4(2 \mu-3 \nu)}\left\{-4+\left(H_{0}^{\mathrm{C}, \mathrm{A}}\right)^{2}(10 \mu+9 \nu)\right\} .
$$

Clearly, when $\operatorname{Re} \xi^{(i)}<0$ for $i=1,2,3$, the corresponding attractor is stable. Since we only consider non-tachyonic matter, eigenvalue (56a) is negative. However, a finite period in which $w<-1$ is not excluded (see e.g.: [47, 48]). If one were to consider other equations of state than the simple linear one $\rho_{\mathrm{M}}=\omega p_{\mathrm{M}}$, this statement might no longer hold [54]. Hence only for $\xi^{(2)}$ when $\Delta>0$, we could encounter a potential instability. Surprisingly, the stability analysis does not depend on the equation of state $\omega$ because $\omega$ enters only through equation (56a). The condition for instability thus reads:

$$
\Delta>\left(\frac{3 H_{0}^{\mathrm{C}, \mathrm{A}}}{2}\right)^{2} .
$$

We can rewrite this equation to find:

$$
4+8 \pi G\left(H_{0}^{\mathrm{C}, \mathrm{A}}\right)^{2}\left(10 b^{\prime}+9 b^{\prime \prime}\right) \gtrless 72 \pi G\left(b^{\prime \prime}-2 b^{\prime} / 3\right)\left(H_{0}^{\mathrm{C}, \mathrm{A}}\right)^{2} .
$$

In the expression above, we should read the inequality $>$ or $<$ whenever $b^{\prime \prime}-2 b^{\prime} / 3>0$ or $b^{\prime \prime}-2 b^{\prime} / 3<0$, respectively. We can now insert either the classical or quantum asymptotes previously derived in equation (29) and verify which of the two above inequalities is satisfied. Upon inserting the expression for the classical attractor, equation (59) yields:

$$
1+32 \pi \lambda b^{\prime} \gtrless 0,
$$

where $\lambda=G \Lambda / 3 \ll 1$ as before. Only the first inequality $>$ will be satisfied. Hence we conclude that the classical attractor is unstable if $b^{\prime \prime}-2 b^{\prime} / 3>0$. The converse will be true if $b^{\prime \prime}-2 b^{\prime} / 3<0$. Likewise, equation (59) for the quantum attractor after some algebra reads:

$$
-1-\frac{32 \pi \lambda}{3} b^{\prime} \gtrless 0,
$$

Concluding, when using $\lambda \ll 1$, we unambiguously find:

$$
\begin{aligned}
& \text { If } b^{\prime \prime}-2 b^{\prime} / 3>0, \quad \text { then }\left\{\begin{array}{l}
\text { Classical attractor unstable } \\
\text { Quantum attractor stable }
\end{array}\right. \\
& \text { If } b^{\prime \prime}-2 b^{\prime} / 3<0, \text { then }\left\{\begin{array}{l}
\text { Classical attractor stable } \\
\text { Quantum attractor unstable }
\end{array}\right.
\end{aligned}
$$

Let us first of all recall that it is precisely the combination $b^{\prime \prime}-2 b^{\prime} / 3$ that multiplies the $\square R$ contribution in the trace anomaly. This calculation thus proves the statements about stability made in e.g. [42] using the Routh-Hurwitz method. Our proof is more general because we include a constant but otherwise arbitrary equation of state parameter $\omega>-1$. Moreover, while the Routh-Hurwitz method can only guarantee stability of a solution (when certain 
determinants are all strictly positive), it does not tell anything about instability [42, 55]. Furthermore appreciate that the singular point in this analysis, $b^{\prime \prime}-2 b^{\prime} / 3=0$, or equivalently $2 \mu / 3-\nu=0$, immediately directs us to the quasi de Sitter spacetime analysis performed in section IVB, where all higher derivative contributions precisely cancel, rendering both attractors stable.

Let us compare figures 7 and 9. In the former figure, we used $b^{\prime \prime}=0$ such that $b^{\prime \prime}-2 b^{\prime} / 3>0$, yielding an unstable classical attractor. However, if $H(0) \leq H_{0}^{\mathrm{C}}$ the quantum anomaly driven asymptote is not an attractor and the Hubble parameter runs away to negative infinity. In the latter figure, we set $b^{\prime \prime}=b^{\prime}$ such that $b^{\prime \prime}-2 b^{\prime} / 3<0$ which gives us a stable classical attractor. Likewise, for initial conditions $H(0) \geq H_{0}^{\mathrm{A}}$ the de Sitter solution is not an attractor and the Hubble parameter rapidly blows up to positive infinity.

In figure 9, we can observe another interesting phenomenon. In this case, the classical attractor is under-damped, resulting in decaying oscillations around the de Sitter attractor. In figure 7 these oscillations are not always present. The eigenvalues (56) develop an imaginary contribution resulting in oscillatory behaviour whenever:

$$
\Delta<0
$$

We thus find:

$$
4+8 \pi G\left(H_{0}^{\mathrm{C}, \mathrm{A}}\right)^{2}\left(10 b^{\prime}+9 b^{\prime \prime}\right) \lessgtr 0
$$

The inequality $<$ or $>$ holds whenever $b^{\prime \prime}-2 b^{\prime} / 3>0$ or $b^{\prime \prime}-2 b^{\prime} / 3<0$ applies, respectively. Again, we verify which of the two inequalities is actually satisfied. To study oscillations around a stable classical attractor, we should take the $>$ inequality (there are no oscillations around an unstable attractor). We thus find:

$$
1+2 \pi \lambda\left(10 b^{\prime}+9 b^{\prime \prime}\right)>0
$$

Clearly, this inequality is always satisfied because $\lambda \ll 1$. We thus conclude that whenever the classical de Sitter attractor is stable, oscillations occur. Furthermore, we can insert the quantum anomaly driven attractor in equation (64). Now, we should use the < inequality in order to study oscillatory behaviour around the (stable) quantum attractor. This yields:

$$
-1-\frac{9}{2} \frac{b^{\prime \prime}}{b^{\prime}}-8 \pi \lambda\left(10 b^{\prime}+9 b^{\prime \prime}\right)<0 .
$$

Oscillatory behaviour around the quantum attractor thus occurs when:

$$
b^{\prime \prime}<-\frac{2}{9} b^{\prime}\left(\frac{1+8 \pi \lambda b^{\prime}}{1+8 \pi \lambda}\right) .
$$

In figure 11 we depicted the parameter space (the $b^{\prime}$ versus $b^{\prime \prime}$ plane) resulting in oscillatory behaviour around the quantum anomaly driven attractor. First of all, we should have $b^{\prime \prime}-2 b^{\prime} / 3>0$ yielding a stable quantum attractor. Of course, one cannot have oscillations around an unstable attractor. Secondly, note that $b^{\prime}<0$ because of equation (4b). Finally, when the newly derived inequality (67) is satisfied, oscillations occur. These considerations divide the phase space into three regions: a region where oscillatory behaviour occurs, another region which results in critically or over-damped behaviour and a part of phase space that is forbidden, as shown in figure 11.

Let us now return to discussing the classical attractor that shows its oscillatory behaviour manifestly. We calculate the frequency of oscillations around this attractor. Taking the square root of (57) and extracting an $i$ we find the (quantum corrected) frequency for oscillations around the classical attractor:

$$
\omega_{\mathrm{C}}=\sqrt{\frac{1+2 \pi \lambda\left(10 b^{\prime}+9 b^{\prime \prime}\right)}{8 \pi G\left(2 b^{\prime} / 3-b^{\prime \prime}\right)}} .
$$

Note that this frequency is independent on the equation of state parameter $\omega$. The frequency of oscillations around the quantum attractor can be found analogously.

Let us analyse the phase space in the case of a stable quantum and classical attractor subsequently. In figure 8 , we visualise the phase space flow for the former case parametrically in the $H(t), \epsilon(t)$ and $\dot{\epsilon}(t)$ directions ${ }^{7}$. The small

\footnotetext{
7 Note we are not able to include the fourth dimension of the phase space, $\ddot{\epsilon}(t)$. However, also $\ddot{\epsilon}(t)$ rapidly approaches zero as time elapses.
} 


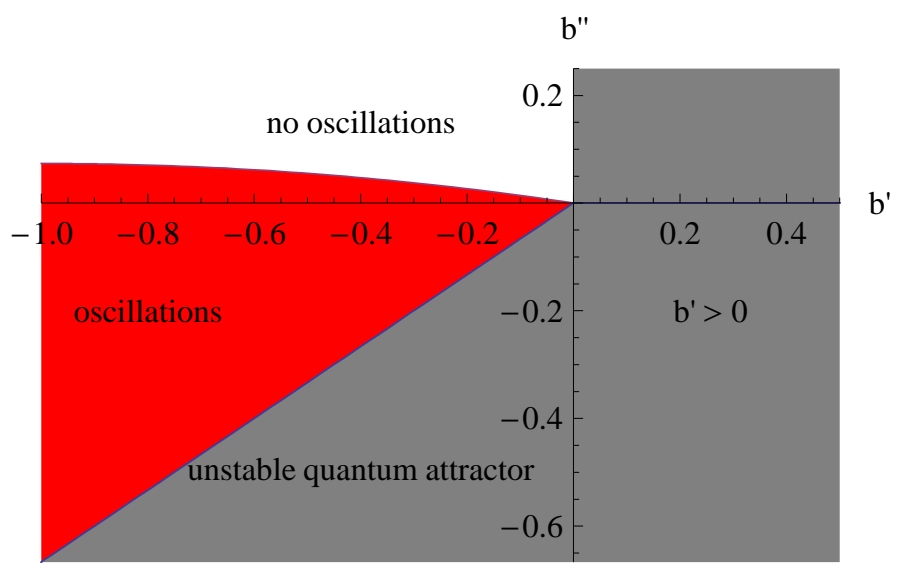

Figure 11: Parameter space for oscillations around the quantum anomaly driven attractor. The gray region (light shaded) is excluded either because $b^{\prime}>0$ or because the quantum attractor has become unstable. The red region (dark shaded) in parameter space shows oscillatory behaviour, whereas the white region is either critically damped or over-damped. We used $\lambda=1 / 50$.

black sphere denotes the quantum anomaly driven attractor. For the latter case, we include in figure 10 the phase space flow for just two initial conditions for $H(t)$ for clarity. The under-damped oscillatory behaviour results in the twister like structure visible in the $H(t), \epsilon(t)$ and $\dot{\epsilon}(t)$ directions.

Finally, we would like to point out that including all higher derivative contributions, which thus corresponds to solving the trace equation exactly, modifies the dynamics of the Hubble parameter significantly. Attractors that were stable in the absence of higher derivatives under certain conditions destabilise. The reason for this, clearly, is attributable to the presence of the $\square R$ term in the trace anomaly, generating these higher derivative contributions. We do not know whether or not incorporating the higher derivatives is a sensible thing to do. Usually higher order derivatives tend to destabilise a system signifying that some particular solutions are not physical. Therefore, in the spirit of Ostrogradsky's theorem, one can question whether the analysis where higher derivative contributions are discarded is correct, or the analysis taking the full trace anomaly into account.

\section{CONCLUSION}

The trace of the Einstein field equations in cosmologically relevant spacetimes together with stress-energy conservation completely captures the dynamics of the Hubble parameter. We have derived the trace anomaly from an effective action in spatially flat FLRW spacetimes. It consists of the local quadratic geometric curvature invariants $R^{2}$ and the Gauss-Bonnet term $E$. Because of counterterms that are supposed to cancel divergences of the as yet unknown underlying fundamental theory, we expect the coefficients in the trace anomaly to change. The physical value of each of these coefficients receives contributions both from the anomalous trace and from these counterterms. Because we do not know the physical value these parameters will take, we must allow them to vary in order to examine all possibilities.

We have studied the dynamics of the Hubble parameter both in quasi de Sitter and in FLRW spacetimes including matter, a cosmological term and the trace anomaly. In quasi de Sitter spacetime, where we restrict the Hubble parameter to vary slowly in time, we find that for various initial conditions $H(t)$ asymptotes either to the classical de Sitter attractor, or to a quantum anomaly driven attractor. We find a region in parameter space where the quantum attractor destabilises. Otherwise, both attractors are stable.

In FLRW spacetimes we include all higher derivative contributions in the trace anomaly. We perform a stability analysis for small perturbations around the two asymptotes. For $b^{\prime \prime}-2 b^{\prime} / 3>0$, the quantum attractor is stable and the classical de Sitter attractor is unstable. On the contrary, for $b^{\prime \prime}-2 b^{\prime} / 3<0$, the quantum attractor is unstable and the de Sitter attractor becomes stable. The singular point in this analysis, $b^{\prime \prime}-2 b^{\prime} / 3=0$, immediately directs us to quasi de Sitter spacetime in which the dynamics is much simpler. In this case, both attractors are stable. The classical de Sitter attractor always shows under-damped oscillatory behaviour and we calculate the frequency of these oscillations. We analyse the phase space of the quantum attractor and conclude there is some region in parameter space for which oscillations occur. 
There is no dynamical effect that influences the effective value of the cosmological constant, i.e.: the classical de Sitter attractor. Based on our semiclassical analysis we thus conclude that the trace anomaly does not solve the cosmological constant problem.

We have studied both the truncated and the exact expression of the trace anomaly in flat FLRW spacetimes. We do not know which of the two approaches is correct. Keeping Ostrogradsky's theorem in mind, higher derivative contributions usually have the tendency to destabilise a dynamical system. Discarding these higher derivatives and studying the trace anomaly in quasi de Sitter spacetime would thus seem plausible.

Finally, one could wonder whether the quantum anomaly driven attractor is physical. The quantum attractor is of the order of the Planck mass $M_{\mathrm{pl}}$, so only when matter in the early Universe is sufficiently dense, $H \simeq \mathcal{O}\left(M_{\mathrm{pl}}\right)$. We then expect to evolve towards the quantum attractor. However, at these early times we also expect perturbative general relativity to break down. Hence, this attractor might even not be there or it may be seriously affected by quantum fluctuations. Quantum fluctuations present at that epoch might even induce tunnelling towards the regime where $H(t)$ asymptotes to the classical attractor.

\section{Acknowledgements}

We thank Tomas Janssen and Jan Smit for useful suggestions. The authors acknowledge financial support from FOM grant 07PR2522 and by Utrecht University.

[1] S. Nobbenhuis, Categorizing Different Approaches to the Cosmological Constant Problem, Found. Phys. 36 (2006) 613 arXiv:gr-qc/0411093.

[2] S. Nobbenhuis, The Cosmological Constant Problem, an Inspiration for New Physics, Ph.D. Thesis, arXiv:gr-qc/0609011.

[3] R. Bousso, TASI Lectures on the Cosmological Constant, [arXiv:hep-th/0708.4231].

[4] J. Donoghue, The Quantum Theory of General Relativity at Low Energies, Helv. Phys. Acta 69 (1996) 269 arXiv:gr-qc/9607039.

[5] J. Donoghue, Perturbative Dynamics of Quantum General Relativity, arXiv:gr-qc/9712070.

[6] N. D. Birrell and P. C. W. Davies, Quantum Fields in Curved Space, Cambridge monographs on Mathematical Physics, Cambridge University Press (1982)

[7] D. M. Capper and M. J. Duff, Trace Anomalies in Dimensional Regularization, Nuovo Cim. A 23, 173 (1974).

[8] S. Deser, M. J. Duff and C. J. Isham, Nonlocal Conformal Anomalies, Nucl. Phys. B 111, 45 (1976).

[9] L. S. Brown, Stress Tensor Trace Anomaly in a Gravitational Metric: Scalar Fields, Phys. Rev. D 15 (1977) 1469.

[10] J. S. Dowker and R. Critchley, The Stress Tensor Conformal Anomaly for Scalar and Spinor Fields, Phys. Rev. D 16 (1977) 3390.

[11] H. S. Tsao, Conformal Anomalies in a General Background Metric, Phys. Lett. B 68 (1977) 79.

[12] M. J. Duff, Observations on Conformal Anomalies, Nucl. Phys. B 125, 334 (1977).

[13] L. S. Brown and J. P. Cassidy, Stress Tensor Trace Anomaly in a Gravitational Metric: General Theory, Maxwell Field, Phys. Rev. D 15 (1977) 2810.

[14] M. J. Duff, Twenty Years of the Weyl Anomaly, Class. Quant. Grav. 11, 1387 (1994) arXiv:hep-th/9308075.

[15] L. H. Ford, Quantum Instability of de Sitter Space-Time, Phys. Rev. D 31 (1985) 710.

[16] I. Antoniadis and E. Mottola, Graviton Fluctuations in de Sitter Space, J. Math. Phys. 32 (1991) 1037.

[17] N. C. Tsamis and R. P. Woodard, The Structure of Perturbative Quantum Gravity on a de Sitter Background, Commun. Math. Phys. 162 (1994) 217.

[18] N. C. Tsamis and R. P. Woodard, Quantum Gravity Slows Inflation, Nucl. Phys. B 474 (1996) 235 arXiv:hep-ph/9602315.

[19] N. C. Tsamis and R. P. Woodard, One Loop Graviton Self-Energy in a Locally de Sitter Background, Phys. Rev. D 54 (1996) 2621 arXiv:hep-ph/9602317.

[20] L. R. W. Abramo, R. H. Brandenberger and V. F. Mukhanov, The Energy-momentum Tensor for Cosmological Perturbations, Phys. Rev. D 56 (1997) 3248 arXiv:gr-qc/9704037.

[21] F. Finelli, G. Marozzi, G. P. Vacca and G. Venturi, Adiabatic Regularization of the Graviton Stress-energy Tensor in de Sitter space-time, Phys. Rev. D 71 (2005) 023522 arXiv:gr-qc/0407101.

[22] A. Bilandzic and T. Prokopec, Quantum Radiative Corrections to Slow-roll Inflation, Phys. Rev. D 76 (2007) 103507 arXiv:0704.1905 [astro-ph]].

[23] T. Janssen and T. Prokopec, A Graviton Propagator for Inflation, Class. Quant. Grav. 25 (2008) 055007 arXiv:0707.3919 $[\mathrm{gr}-\mathrm{qc}]]$.

[24] T. Janssen and T. Prokopec, Implications of the Graviton One-loop Effective Action on the Dynamics of the Universe, arXiv:0807.0447 [gr-qc].

[25] T. Janssen, S. P. Miao and T. Prokopec, Graviton One-loop Effective Action and Inflationary Dynamics, arXiv:0807.0439 $[\mathrm{gr}-\mathrm{qc}]$.

[26] N. Bilic, B. Guberina, R. Horvat, H. Nikolic and H. Stefancic, On Cosmological Implications of Gravitational Trace Anomaly, Phys. Lett. B 657 (2007) 232 [arXiv:gr-qc/0707.3830].

[27] R. Schutzhold, Small Cosmological Constant from the QCD Trace Anomaly?, Phys. Rev. Lett. 89 (2002) 081302. 
[28] E. T. Tomboulis, Dynamically Adjusted Cosmological Constant and Conformal Anomalies, Nucl. Phys. B 329 (1990) 410.

[29] I. Antoniadis and E. Mottola, 4-D Quantum Gravity in the Conformal Sector, Phys. Rev. D 45 (1992) 2013.

[30] I. Antoniadis, Dynamics of the Conformal Factor in 4-D Gravity, arXiv:hep-th/9211055].

[31] I. Antoniadis, P. O. Mazur and E. Mottola, Fractal Geometry of Quantum Spacetime at Large Scales, Phys. Lett. B 444 (1998) 284 arXiv:hep-th/9808070.

[32] H. Salehi and Y. Bisabr, Conformal Anomaly and Large Scale Gravitational Coupling, Int. J. Theor. Phys. 39 (2000) 1241 arXiv:hep-th/0001095.

[33] I. Antoniadis, P. O. Mazur and E. Mottola, Cosmological Dark Energy: Prospects for a Dynamical Theory, New J. Phys. 9 (2007) 11 arXiv:gr-qc/0612068.

[34] R. J. Riegert, A Nonlocal Action for the Trace Anomaly, Phys. Lett. B 134, 56 (1984).

[35] E. Komatsu et al. [WMAP Collaboration], Five-Year Wilkinson Microwave Anisotropy Probe (WMAP) Observations: Cosmological Interpretation, arXiv:0803.0547 [astro-ph].

[36] A. A. Starobinsky, A New Type of Isotropic Cosmological Models without Singularity, Phys. Lett. B 91 (1980) 99.

[37] S. W. Hawking, T. Hertog and H. S. Reall, Trace Anomaly Driven Inflation, Phys. Rev. D 63 (2001) 083504 arXiv:hep-th/0010232.

[38] J. C. Fabris, A. M. Pelinson and I. L. Shapiro, Anomaly-induced Effective Action and Inflation, Nucl. Phys. Proc. Suppl. 95, 78 (2001) arXiv:hep-th/0011030.

[39] I. L. Shapiro, An Overview of the Anomaly-induced Inflation, Nucl. Phys. Proc. Suppl. 127 (2004) 196 arXiv:hep-ph/0311307.

[40] I. L. Shapiro, Effective Field Theory and Fundamental Interactions, arXiv:hep-th/0412115.

[41] I. L. Shapiro and J. Sola, A Modified Starobinsky's Model of Inflation: Anomaly-induced Inflation, SUSY and Graceful Exit, arXiv:hep-ph/0210329.

[42] A. M. Pelinson, I. L. Shapiro and F. I. Takakura, On the Stability of the Anomaly-induced Inflation, Nucl. Phys. B 648, 417 (2003) arXiv:hep-ph/0208184.

[43] A. M. Pelinson, I. L. Shapiro and F. I. Takakura, Stability Issues in the Modified Starobinsky Model, Nucl. Phys. Proc. Suppl. 127, 182 (2004) arXiv:hep-ph/0311308.

[44] I. Brevik and J. Quiroga Hurtado, Vanishing Cosmological Constant in Modified Gauss-Bonnet Gravity with Conformal Anomaly, Int. J. Mod. Phys. D 16 (2007) 817 arXiv:gr-qc/0610044.

[45] R. P. Woodard, Avoiding Dark Energy with 1/R Modifications of Gravity, Lect. Notes Phys. 720 (2007) 403 arXiv:astro-ph/0601672.

[46] M. V. Fischetti, J. B. Hartle and B. L. Hu, Quantum Effects in the Early Universe. 1. Influence of Trace Anomalies on Homogeneous, Isotropic, Classical Geometries, Phys. Rev. D 20 (1979) 1757.

[47] V. K. Onemli and R. P. Woodard, Quantum Effects can Render $w<-1$ on Cosmological Scales, Phys. Rev. D 70 (2004) 107301 arXiv:gr-qc/0406098.

[48] E. O. Kahya and V. K. Onemli, Quantum Stability of a $w<-1$ Phase of Cosmic Acceleration, Phys. Rev. D 76 (2007) 043512 arXiv:gr-qc/0612026.

[49] I. L. Shapiro, Local Conformal Symmetry and its Fate at Quantum Level, PoS IC2006 (2006) 030 arXiv:hep-th/0610168.

[50] I. L. Shapiro, Effective Action of Vacuum: Semiclassical Approach, Class. Quant. Grav. 25, 103001 (2008) arXiv:0801.0216 $[\mathrm{gr}-\mathrm{qc}]]$.

[51] P. C. W. Davies, Singularity Avoidance and Quantum Conformal Anomalies, Phys. Lett. B 68 (1977) 402.

[52] E. V. Gorbar and I. L. Shapiro, Renormalization Group and Decoupling in Curved Space, JHEP 0302 (2003) 021 arXiv:hep-ph/0210388.

[53] E. V. Gorbar and I. L. Shapiro, Renormalization Group and Decoupling in Curved Space. II: The Standard Model and Beyond, JHEP 0306 (2003) 004 arXiv:hep-ph/0303124.

[54] S. Nojiri, S. D. Odintsov and S. Tsujikawa, Properties of Singularities in (Phantom) Dark Energy Universe, Phys. Rev. D 71 (2005) 063004 arXiv:hep-th/0501025.

[55] E. W. Weisstein, "Routh-Hurwitz Theorem.", From MathWorld - A Wolfram Web Resource, http://mathworld.wolfram.com/Routh-HurwitzTheorem.html. 